\title{
A Fine-Grained Analysis of Misinformation in COVID-19 Tweets
}

\section{Sumit Kumar ( $\nabla$ sumit.atlancey@gmail.com )}

Birla Institute of Technology, Mesra, India

\section{Raj Ratn Pranesh}

Birla Institute of Technology, Mesra, India

\section{Kathleen M. Carley}

Carnegie Mellon University, Pittsburgh PA, USA

\section{Research Article}

Keywords: Misinformation, Classification, Transformer

Posted Date: June 7th, 2021

DOl: https://doi.org/10.21203/rs.3.rs-588650/v1

License: (c) (i) This work is licensed under a Creative Commons Attribution 4.0 International License. Read Full License 


\title{
A Fine-Grained Analysis of Misinformation in COVID-19 Tweets
}

\author{
Sumit Kumar*1, Raj Ratn Pranesh ${ }^{\star 2}$, and Kathleen M. Carley ${ }^{3}$ \\ 1 Birla Institute of Technology, Mesra, India \\ sumit.atlancey@gmail.com \\ 2 Birla Institute of Technology, Mesra, India \\ raj.ratn18@gmail.com \\ 3 Carnegie Mellon University, Pittsburgh PA, USA \\ kathleen.carley@cs.cmu.edu
}

\begin{abstract}
In the past few years, there has been an expeditious growth in the usage of social media platforms and blogging websites which has passed 3.8 billion marks of active users that use text as a prominent means for interactive communication. A fraction of users spread misinformation on social media. As Twitter has 330 million monthly active users, researchers have been using it as a source of data for misinformation identification [1]. In this paper, we have proposed a Twitter dataset for fine-grained classification. Our dataset is consist of 1970 manually annotated tweets and is categorized into 4 misinformation classes, i.e, "Irrelevant", "Conspiracy", "True Information", and "False Information" based on response erupted during COVID-19. In this work, we also generated useful insights on our dataset and performed a systematic analysis of various language models, namely, $R N N$ (BiLSTM, LSTM), $C N N$ (TextCNN), BERT, ROBERTA and ALBERT for the classification task on our dataset. Through our work, we aim at contributing to the substantial efforts of the research community for the identification and mitigation of misinformation on the internet.
\end{abstract}

Keywords: Misinformation · Classification · Transformer.

\section{Introduction}

The global outbreak of COVID-19 resulted in a crisis all around the world. The pandemic originated from Wuhan, China, and rapidly spread all over the world causing casualties and affected human life drastically. By November 1, 2020, the total case count crossed the bar of 46.2 Million, and 1.2 Million people have lost their lives worldwide. As novel coronavirus continues to spread, it has a massive impact on several sectors for instance country economy, public and private sectors, government bodies, and above all affecting the mental and physical health of the people by tempering their daily routines. In spite of this, people expressed

\footnotetext{
* equal contribution
} 
their thoughts, news, opinions, and information related to COVID-19 across several social media platforms such as Twitter, Facebook, Whatsapp, Instagram, and Reddit. We have attempted to track this panic response through Twitter. An enormously large number of tweets were tweeted during the outbreak of novel coronavirus. The tweets of our interests are discussed conspiracy theories related to the disease, true prevention and cures, and fake prevention and cures. Studies have shown that numerous people connect to the internet and social media platforms every day to gather information/news through them. As in [2] a great amount of human-generated information being exchanged every day, it has attracted researchers to explore, analyze, and generate valuable insights about people reaction to COVID-19 to identify and mitigate through misinformation detection. The sudden rise in the flow of COVID-19 related information on the internet has caused perturbing concerns towards the risk of social cybersecurity. According to the paper [3], thousands of articles showcasing COVID-19 disinformation such as deception based on bogus cures and strategies of prevention, false portrayal of government policies, and virus spread have engendered conspiracy theories throughout the social-economic sectors of society. In this work, we took a step toward solving a very crucial problem of detecting and mitigating COVID-19 related misinformation present on the social media platforms such as Twitter. We have manually collected and annotated 1970 user-generated COVID-19 tweets into 4 classes of misinformation. We have also performed a comparative analysis of various existing language models for the misinformation classification task.

The four key motivation in this paper are:

- With the advancement of technology, digital news is more widely exposed to users globally and contributes to the increment of spreading hoaxes and disinformation online.

- COVID-19 being one of the largest recent pandemic, the spread of misinformation related to COVID-19 can make the current situation worse and eventually adversely affect the overall functioning of daily human life.

- Hence, it is very crucial for us to develop a misinformation detection system that would analyze the content (semantic approach) of user-generated data such as tweets and assign a most probable misinformation class to it.

- Developing a misinformation classification system would help in the identification and filtration of information distributed all over the social media platforms and therefore, in the future automatic misinformation systems can be deployed for monitoring and mitigation of misinformation.

Few challenges that we faced are:

- The most challenging task is to determine the type of misinformation in a tweet which could be difficult for humans to trace and distinguish themselves, let alone machines.

- COVID-19 is a recent occurrence. So, because of the lack of standardized datasets on the internet, we have to manually annotate to prepare data for training. 


\section{Related Work}

In the past few years, Research has been done in natural language processing, which involves analyzing and identification of misinformation in textual representations.

In the paper [1], the author made use of Twitter data for a detailed analysis of Tweets mentioning "fake news" URLs and disinformation story-lines that are most likely to be spread by regular users. They also showed that unlike actual news and normal tweets, tweets containing URLs pointing to "fake news" sites are most likely to be retweeted within the source country and so are less likely to spread internationally. Along with that, They used machine learning systems to predict users' latent attributes, such as their locations and political orientations.

In the paper [4], the author presented a method named twitter_sim ABM designed for exploring the explicit actions users make in Twitter, which captures the varied actions of malicious agents like bots/tolls. They showed the use of this model in exploring the emerging behavior of specific disinformation maneuvers. Also, they validated some key variables in the model from empirical Twitter data.

Moreover, the author in the paper [5] deliberately tried to discuss some key points such as misinformation detection from text classification. They utilized feature engineering methods and sources information using the dataset, they successfully explain the identification of misinformation spreaders and how they propagate the information.

The above-summarised work talks about various methods for misinformation identification and detection using a machine learning model. These papers performed the misinformation classification task on limited data with very general classes. While our paper focuses on various deep learning language models that were trained on our proposed dataset with fine-grained misinformation classes.

\section{Dataset}

This section will explain the dataset generation process and description of the dataset that we proposed in this paper. We condense the approach for collecting and Pre-processing the user-generated dataset through the tweets to come up with a final dataset. We have summarised the features of the dataset through some examples in Table 3, along with the data annotation schemes and guidelines.

\subsection{Data Collection}

We crawled through Twitter data using the Tweepy ${ }^{4}$ which is a Python library for accessing Twitter Application Programming Interface $\left(\mathrm{API}^{5}\right)$, and collected a sample of tweets.

\footnotetext{
4 https://www.tweepy.org/

${ }^{5}$ https://developer.twitter.com/en/docs/twitter-api/v1/tweets/search/apireference/get-search-tweets
} 
To extract the required tweets, we build a set of keywords related to the usage of hashtags in the semantic sentence (e.g., \#COVID-19, \#Coronavirus) in both lowercase and uppercase. The following keywords such as covid19, quarantine, quarantinelife, publichealth, pandemic, terrorism, BioWeapon, immune, 5G, wuhan, whancoronavirus, conspiracytheory were used to collect tweets. Hence, the final collected dataset contains 1970 tweets.

\begin{tabular}{|c|c|}
\hline Class & tweet count \\
\hline Irrelevant & 768 \\
\hline Conspiracy & 468 \\
\hline True Information & 462 \\
\hline False Information & 272 \\
\hline Total & 1970 \\
\hline
\end{tabular}

Table 1. Class distribution of dataset

\begin{tabular}{|c|c|}
\hline Name & Annotation Class \\
\hline Mention of Irrelevant text & 0 \\
\hline Mention of Conspiracy text & 1 \\
\hline Mention of True Information text & 2 \\
\hline Mention of False Information text & 3 \\
\hline
\end{tabular}

Table 2. Label associated with each class

\subsection{Data Annotation}

The gathered data were annotated by two human annotators of linguistic background and proficiency in English using the categories mentioned in Table 2. We chose the categories based on the frequency of the occurrences of Irrelevant text, Conspiracy text, True Information text, and False Information text associated with tweets.

A general description of each class is given below, as in [6]. We used a codebook for a detailed description of each class. The codebook is available on zenodo and can be accessed using this link ${ }^{6}$.

Mention of Irrelevant text: A tweet is categorized as irrelevant if COVID-19 or SARS-CoV-2 may or may not be specified, but if it cannot be classified in any of the other categories listed below.

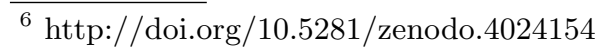


Mention of Conspiracy text: A tweet, whether it endorses a conspiracy theory, must be labeled as a conspiracy. Some examples of COVID-19 based conspiracy themes include:

- It is a bio-weapon.

- Electromagnetic fields and the development of cellular 5G technology have contributed to outbreaks of COVID-19.

- It leaked from the Wuhan Labs or Wuhan Institute of Virology in China.

- Dean Koontz had expected it.

Mention of True Information text: A tweet shall be listed as true information if it endorses the pain-relieving care approach (rest and sleep, stay warm, drink lots of water, etc.) and if it specifically endorses the preventive method and if one of the following conditions is fulfilled:

- The details were checked via the website of the World Health Organisation (WHO) or the Centre for Disease Control and Prevention (CDC).

- A peer-reviewed scientific journal that appears as both 'Active' and 'Refereed/Peerreviewed' in Ulrich's Global Serials Directory supports the details.

- Tweet ties directly to news articles that a peer-reviewed research article references accurately.

Mention of False Information text: If the text endorses a cure and all the following requirements are met, a tweet is labeled as false information:

- The World Health Organisation (WHO) site or the Centre for Disease Control and Prevention (CDC) site cannot verify the facts.

- A peer-reviewed scientific journal that appears as both 'Active' and 'Refereed/Peerreviewed' in Ulrich's Global Serials Directory does not support the data.

- The evidence calls out a false cure, fake mitigation, fake therapy, or a conspiracy theory or makes fun of it.

\subsection{Dataset Preprocessing}

Before conducting the analysis and experiments, we preprocessed tweets by firstly converting them to lowercase representation. We also made the tweets free from any unnecessary elements such as username, mentions, links, retweets. We used NLTK ${ }^{7}$, a Python module for text processing that removed the English stop words and performed lemmatization of tweets.

\section{Methodology}

In this section, we have sequentially discussed the architecture of various classification models used in our experiment. We used LSTM, Bi-LSTM, TextCNN, $C N N-R N N, R N N-C N N$, Distil_Bert, BERT $T_{\text {base }}, B E R T_{\text {large }}$, Distil_RoBERTa,

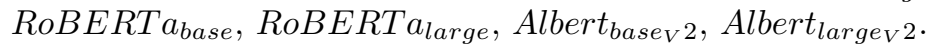

\footnotetext{
7 https://www.nltk.org/
} 


\begin{tabular}{|l|l|}
\hline Class & Tweet Data \\
\hline Irrelevant & $\begin{array}{l}\text { Morning everyone. I hope you all have a relaxing Sunday! } \\
\text { \#Stay AtHomeSaveLives \#coronavirus \#covid19 \#SundayThoughts }\end{array}$ \\
\hline Irrelevant & $\begin{array}{l}\text { Did you know that } 66 \% \text { of U.S. workers are working remotely } \\
\text { full-time as a result of the \#coronavirus pandemic. }\end{array}$ \\
\hline Conspiracy & $\begin{array}{l}\text { Many people across the globe lost lives due to \#coronavirus } \\
\text { spreaded by \#China. It is not a disease, it is a biological } \\
\text { weapon created by China to destroy and rule the world. } \\
\text { Why there are no cases in China, while being the } \\
\text { most populated country across the globe }\end{array}$ \\
\hline Conspiracy & $\begin{array}{l}\text { Covid 19 is a genetically modified virus. It is a bio weapon that came } \\
\text { from a bio lab in China and has nothing to do with eating meat. } \\
\text { It did not come from eating bat soup and it is spread by } \\
\text { respiratory droplets NOT what you eat. }\end{array}$ \\
\hline True Information & $\begin{array}{l}\text { The French Ministry of Health has issued a press release to } \\
\text { notify all citizens that cocaine does NOT protect } \\
\text { against COVID-19. }\end{array}$ \\
\hline True Information & $\begin{array}{l}\text { You took it to protect against malaria. There are NO trials } \\
\text { that show it is effective against \#COVID19 }\end{array}$ \\
\hline False Information & $\begin{array}{l}\text { Gargle with Listerine. Preferably the alcohol Listerine. } \\
\text { It will kill the covid-19 in your throat and mouth. }\end{array}$ \\
\hline False Information & $\begin{array}{l}\text { Use the space heater or blow dryer and breathe } \\
\text { the heat in through your nose. It will kill the covid-19 in } \\
\text { your nostrils. Use a pinch of salt in boiling water breathe in. }\end{array}$ \\
\hline
\end{tabular}

Table 3. Tweet with their respective misinformation class 


\section{$4.1 \mathrm{CNN}$}

In this subsection, we described the Convolution Neural Networks [7] for classification and also outlines the methods for text classification specifically. Convolutional neural networks are multistage trainable neural network architectures developed for classification tasks [8]. Each stage contains different layers as summarized below:

- Embedding Layer: The function of an embedding layer is to transform the text inputs into a form that can be used by the CNN model. Here, each word of a text document is transformed into a dense vector of fixed size.

- Convolutional Layer: A Convolutional layer comprises of several kernel matrices that perform the convolution mathematical operation on their input and process an output matrix of features upon the addition of a bias value.

- Pooling Layer: A pooling layer performs dimensionality reduction of the input feature vectors. It uses sub-sampling to the output of the convolutional layer matrices combing neighboring elements. we have used the max-pooling function for the pooling.

- Fully Connected Layer: : A classic fully connected neural network layer is connected to the Pooling layers via a Dropout layer in order to prevent overfitting. The softmax activation function is used for defining the final output of this layer. The following objective function is commonly used in the task:

$$
E_{w}=\frac{1}{n} \sum_{p=1}^{P} \sum_{j=1}^{N_{l}}\left(o_{j, p}^{L}-o_{j, p}\right)^{2}
$$

where $\mathrm{P}$ is the number of patterns, $o_{j, p}^{L}$ is the output of $j^{\text {th }}$ neuron that belongs to $L^{t h}$ layer, $N_{l}$ is the number of neurons in output of $L^{t h}$ layer, $y_{j, p}$ is the desirable target of $j^{t h}$ neuron of pattern $\mathrm{p}$ and $y_{i}$ is the output associated with an input vector $x_{i}$ to the CNN.

In order to minimize the cost function $E_{w}$, we use Adam Optimizer [9].

\subsection{RNN}

Recurrent neural networks (RNN) have been used to produce promising results on different tasks, along with language model and speech recognition $[10,11]$. An RNN predicts the current output conditioned on long-distance features by keeping a memory based on previous information.

An input layer represents features at time t. One-hot vectors for words, dense vector features such as word embeddings, or sparse features usually represent an input layer. An input layer has the same dimensionality as feature size. An output layer represents a probability distribution over labels at time $t$ and also has the same dimensionality as the size of the labels. Compared to the feed forward network, an RNN holds a relation between the previous hidden state and the current hidden state. This relation is made through the recurrent layer which 
stores history information. The following equation is used to calculate the values in the hidden and output layers:

$$
\begin{gathered}
\mathbf{h}(t)=f(\mathbf{U x}(t)+\mathbf{W h}(t-1)) \\
\mathbf{y}(t)=g(\mathbf{V h}(t))
\end{gathered}
$$

where $\mathrm{U}, \mathrm{W}$, and $\mathrm{V}$ are the connection weights to be computed during training, and $f(z)$ and $g(z)$ are sigmoid and activation functions as given below:

$$
\begin{aligned}
& f(z)=\frac{1}{1+e^{-z}} \\
& g\left(z_{m}\right)=\frac{e^{z_{m}}}{\sum_{k} e_{k}^{z}}
\end{aligned}
$$

For the purpose of sequence tagging, we used Long Short Term Memory (LSTM) and Bidirectional Long Term Short Memory (Bi-LSTM) as in [12, 11, $13]$.

LSTM networks use purpose-built memory cells to update the hidden layer values. Therefore, they may perform better at finding and utilizing long-range dependencies in the data, unlike a standard RNN. The following equation implements the LSTM model:

$$
\begin{gathered}
i_{t}=\sigma\left(W_{x i} x_{t}+W_{h i} h_{t-1}+W_{c i} c_{t-1}+b_{i}\right) \\
f_{t}=\sigma\left(W_{x f} x_{t}+W_{h f} h_{t-1}+W_{c f} c_{t-1}+b_{f}\right) \\
o_{t}=\sigma\left(W_{x o} x_{t}+W_{h o} h_{t-1}+W_{c o} c_{t}+b_{o}\right) \\
h_{t}=o_{t} \tanh \left(c_{t}\right)
\end{gathered}
$$

For a given time, both past and future input features can be accessed in the sequence tagging task. Therefore, we can also utilize a bidirectional LSTM network (Bi-LSTM) as proposed by the author [13].

\subsection{CNN-RNN}

For designing a CNN-RNN classification model, we stacked the CNN layer over the RNN layer. Firstly, the embedding layer of dimension 300 is utilized to generate a vector representation of tweet text using GloVe [14]. The embeddings are then supplied into a spatial dropout layer $(\mathrm{p}=0.4)$, followed by a convolution layer and a max-pooling layer ( pool size $=2$ ). The polled output is then supplied into a BiLSTM layer followed by a global max polling layer. Finally, the obtained polled output is then passed into a dense layer with 256-dimension (with Relu activation[15]), and then the prediction is made using a d layer with softmax activation. 


\subsection{RNN-CNN}

For designing an RNN-CNN classification model, we employed a single BiLSTM layer over the top of a 1D-CNN layer in the following way. The generated embeddings from the embedding layer after applying spatial dropout $(\mathrm{p}=0.4)$ were supplied into a BiLSTM with rnn units $=300$, followed by a convolution layer with dimension $=64$. Over the obtained output, the global average pool and global max pool values were extracted and concatenated to produce sentencelevel embedding which is then passed to a dense layer with Relu activation[15]. Finally, a hidden layer of size 4 with a softmax function is used to make final predictions.

\subsection{Transformer Language Models}

Large pre-trained language models such as BERT, RoBERTa, ALBERT have gained a lot of popularity in NLP. These language models have been shown to learn remarkably well on downstream tasks including machine translation, question-answering, text classification, and summarization.

In our work, we have utilized (i) three variant of BERT[16] model: Distil_Bert, BERT $T_{\text {base }}, B E R T_{\text {large }}$, (i) three variant of RoBERTa[17] model: Distil_RoBERTa, RoBERTa $a_{\text {base }}$, RoBERT $a_{\text {large }}$ and tho variant of ALBERT[18]: Albert $_{\text {base }_{V} 2}$, Albert large $_{V} 2$. Transformers are contextualized word presentation model, pre-trained using bidirectional transformers [19]. Basically, each model uses the work for predicting the next sentence and thus learns the embeddings with a larger context. The transformer architectures such as BERT, RoBERTa, and $A L B E R T$ were needed to be fine-tuned for the misinformation classification task. We use a pool of labeled training examples for fine-tuning BERT for misinformation detection task using the balanced set of proposed annotated data. We performed the fine-tuning of each pretrained language model by building a custom classification head on top of the models. The classification head was consist of a dropout layer $(\mathrm{p}=0.05)$ followed by a linear layer $(\operatorname{size}=768)$ with Mish[20] activation function, followed by another dropout layer and a final linear layer $($ size $=768)$. The averaged pool of sequential output from 12 encoding layers of used as the custom classifier head's input.

\section{Result and Discussion}

In this section, we have summarised the result obtained in our experiment and also discussed the performance of various classification models on our dataset. As seen in the table 4, the RoBERTa-large performs better than the rest of the models having an F1-score of $76 \%$ with the precision of $73.75 \%$ and recall of $73.5 \%$ on the dataset. Since RoBERTa-large is trained on a bigger corpus compared with the training datasets of other models, and the1 optimized hyperparameters make this model more suitable for the task. Distil-Roberta model had a precision of $71 \%$, this makes this model more favorable in detecting true informative 


\begin{tabular}{|l|c|c|c|}
\hline ModelsScore & Precision & Recall & F1 \\
\hline LSTM & 70.78 & 63.46 & 66.87 \\
\hline Bi-LSTM & 70.67 & 65.23 & 67.87 \\
\hline TextCNN & 70.33 & 66.26 & 65.74 \\
\hline CNN-RNN & 71.84 & 70.98 & 71.40 \\
\hline RNN-CNN & 70.15 & 67.41 & 68.75 \\
\hline AlBERT-large-v2 & 58.75 & 58.25 & 58.00 \\
\hline AlBERT-base-v2 & 55.20 & 54.90 & 55.23 \\
\hline BERT-large & 67.00 & 66.75 & 69.00 \\
\hline BERT-base & 66.5 & 66.25 & 68.00 \\
\hline Distil-BERT & 66.2 & 66.00 & 65.00 \\
\hline RoBERTa-base & 77.5 & 75.9 & 74.00 \\
\hline Distil-RoBERTa & 71.00 & 71.25 & 71.00 \\
\hline RoBERTa-large & 73.75 & 73.5 & 76.00 \\
\hline
\end{tabular}

Table 4. Performance score (in \%) of various models

tweets. BERT-large and BERT-base had a comparatively better result on the dataset, making these models suitable for the task. As for the Distil-BERT, because of fewer parameters, it was hard for this model to infer in favor of this task. As for the Albert-large, it outperformed its smaller version of the model by $2.77 \%$ F1-score. Moving onto the untrained networks, the CNN-RNN model comparatively performed better than the other networks, this is because of the CNN architecture, as it is able to highlight the calculated value using kernels, whereas for the RNN-CNN model it surpassed the TextCNN model by $3.01 \%$. LSTM and Bi-LSTM model was also great for the task as they scored $66.87 \%$ and $67.87 \%$ F1-score respectively.

\section{Conclusion}

In this paper, we have presented a study of COVID-19 misinformation in tweets. We manually created a dataset consisting of 1970 tweets annotated in 4 classes of misinformation. We utilized various deep learning language models such as RNNs, CNN, BERT, RoBERTa, ALBERT, and performed a comparative analysis of these models for the misinformation type classification task. In our study, we found that the larger pretrained model RoBERTa performed better than the other models. We strongly believe that our model can help infiltration of misinformation data present on the internet and to understand public perceptions during the pandemic. In the future, we aim at collecting more annotated training data for improving our model's robustness and contextual understanding for better performance in the classification task. Task such as discovering topics and extracting keywords from multilingual tweets would be interesting. 


\section{References}

1. Huang, Binxuan, and Kathleen M. Carley. "Disinformation and Misinformation on Twitter during the Novel Coronavirus Outbreak." arXiv preprint arXiv:2006.04278 (2020)

2. Matsa, K.E. and Shearer, E., 2018. News use across social media platforms 2018. Pew Research Center, 10.

3. Carley, K.M., 2020. Social cybersecurity: an emerging science. Computational and Mathematical Organization Theory, 26(4), pp.365-381.

4. Beskow, D.M. and Carley, K.M., 2019, December. Agent based simulation of bot disinformation maneuvers in Twitter. In 2019 Winter Simulation Conference (WSC) (pp. 750-761). IEEE.

5. Wu, L., Morstatter, F., Carley, K.M. and Liu, H., 2019. Misinformation in social media: definition, manipulation, and detection. ACM SIGKDD Explorations Newsletter, 21(2), pp.80-90.

6. Memon, S.A. and Carley, K.M., 2020. Characterizing covid-19 misinformation communities using a novel twitter dataset. arXiv preprint arXiv:2008.00791.

7. Fukushima, K., 1988. Neocognitron: A hierarchical neural network capable of visual pattern recognition. Neural networks, 1(2), pp.119-130.

8. LeCun, Y., Bottou, L., Bengio, Y. and Haffner, P., 1998. Gradient-based learning applied to document recognition. Proceedings of the IEEE, 86(11), pp.2278-2324.

9. Kingma, D.P. and Ba, J., 2014. Adam: A method for stochastic optimization. arXiv preprint arXiv:1412.6980.

10. Kombrink, S., Mikolov, T., Karafiát, M. and Burget, L., 2011. Recurrent neural network based language modeling in meeting recognition. In Twelfth annual conference of the international speech communication association.

11. Graves, A. and Schmidhuber, J., 2005. Framewise phoneme classification with bidirectional LSTM and other neural network architectures. Neural networks, 18(5-6), pp.602-610.

12. Hochreiter, S. and Schmidhuber, J., 1997. Long short-term memory. Neural computation, 9(8), pp.1735-1780.

13. Graves, A., Mohamed, A.R. and Hinton, G., 2013, May. Speech recognition with deep recurrent neural networks. In 2013 IEEE international conference on acoustics, speech and signal processing (pp. 6645-6649). IEEE.

14. Pennington, J., Socher, R. and Manning, C.D., 2014, October. Glove: Global vectors for word representation. In Proceedings of the 2014 conference on empirical methods in natural language processing (EMNLP) (pp. 1532-1543).

15. Nair, V. and Hinton, G.E., 2010, January. Rectified linear units improve restricted boltzmann machines. In ICML.

16. Devlin, J., Chang, M.W., Lee, K. and Toutanova, K., 2018. Bert: Pre-training of deep bidirectional transformers for language understanding. arXiv preprint arXiv:1810.04805.

17. Liu, Y., Ott, M., Goyal, N., Du, J., Joshi, M., Chen, D., Levy, O., Lewis, M., Zettlemoyer, L. and Stoyanov, V., 2019. Roberta: A robustly optimized bert pretraining approach. arXiv preprint arXiv:1907.11692.

18. Lan, Z., Chen, M., Goodman, S., Gimpel, K., Sharma, P. and Soricut, R., 2019. Albert: A lite bert for self-supervised learning of language representations. arXiv preprint arXiv:1909.11942.

19. Vaswani, A., Shazeer, N., Parmar, N., Uszkoreit, J., Jones, L., Gomez, A.N., Kaiser, Ł. and Polosukhin, I., 2017. Attention is all you need. Advances in neural information processing systems, 30, pp.5998-6008. 
12 Sumit Kumar, Raj Ratn Pranesh^, and Kathleen M. Carley

20. Misra, D., 2019. Mish: A self regularized non-monotonic neural activation function. arXiv preprint arXiv:1908.08681. 


\section{Supplementary Files}

This is a list of supplementary files associated with this preprint. Click to download.

- MisinformationNLPBaseline.ipynb

- Screenshot20210603at10.10.37PM.png

- Screenshot20210603at10.10.54PM.png

- README.md

- MisinformationNLPBaseline.ipynb 